\section{International Scientific Journal Theoretical \& Applied Science}

\author{
p-ISSN: 2308-4944 (print) e-ISSN: 2409-0085 (online) \\ Year: $2015 \quad$ Issue: $05 \quad$ Volume: 25
}

Published: $30.05 .2015 \quad$ http://T-Science.org
Svetlana Valeryevna Fedorova

Candidate of Technical Sciences, associate professor

Irkutsk National Research Technical University,

Russia

fsta@istu.irk.ru

SECTION 12. Geology. Anthropology. Archeology.

\title{
OPTIMIZATION OF TECHNOLOGICAL PROCESSES OF PREPARATION OF RAW MATERIALS FOR PRODUCTION OF ELECTRIC HEATERS
}

\begin{abstract}
The problem of waste-free use of mineral raw materials gains the big importance for a national economy. And the micalex on the basis of which scientific researches were conducted, is rather new composite material finding the increasing distribution in modern equipment, everything deeper taking root into area of high temperatures.

Key words: raw materials, waste-free technologists, composite materials, electric heaters.

Language: Russian

Citation: Fedorova SV (2015) OPTIMIZATION OF TECHNOLOGICAL PROCESSES OF PREPARATION OF RAW MATERIALS FOR PRODUCTION OF ELECTRIC HEATERS. ISJ Theoretical \& Applied Science 05 (25): 14-18.

Soi: http://s-o-i.org/1.1/TAS*05(25)4 Doi: crossef http://dx.doi.org/10.15863/TAS.2015.05.25.4

\section{ОПТИМИЗАЦИЯ ТЕХНОЛОГИЧЕСКИХ ПРОЦЕССОВ ПОДГОТОВКИ СЫРЬЯ ДЛЯ ПРОИЗВОДСТВА ЭЛЕКТРОНАГРЕВАТЕЛЕЙ}

\begin{abstract}
Аннотация: Проблема безотходного использования минерального сырья приобретает большую значимость для народного хозяйства. А микалекс, на основе которого проводились научные исследования, является сравнительно новым композиционнымм материалом, находящим все большее распространение в современной технике, все глубже внедряющийся в область высоких температур.
\end{abstract}

Ключевые слова: сырье, безотходные технологи, композиционные материалы, электронагреватели.

С целью утилизации промышленных отходов, как стекольной промышленности, так и некондиционного слюдяного сырья, было предложено следующее техническое решение. Нами был разработан вариант технологии производства более выгодного в экономическом отношении нагревателя, составной частью, которого является стеклосвязка, полученная на основе отходов - стекольного производства с добавками борной кислоты $\mathrm{H}_{3} \mathrm{BO}$, азотнокислого бария $\mathrm{Ba}(\mathrm{NO} 3) 3 . \quad$ кремнефтористого натрия $\mathrm{Na}_{2} \mathrm{SiF}_{6}$, азотнокислого калия $\mathrm{KNO}_{3}$ и мелкоразмерной слюды мусковит. Отходы стекольной промышленности дробились на щековой дробилке, с целью получения выхода равномерных кусков стеклоотходов. Процесс дробления проходил в аппарате с верхним подвесом подвижной щеки и вертикальным шатуном. Работа данной конструкции обеспечивала бесперебойный выход раздробленного стекла. В процессе работы было определено, что дробление можно завершить по прошествию первой стадии, так как при завершении процесса дробления удалось получить стекло крупностью до 30 мм. Стекло является довольно хрупким материалом, в его аморфной структуре имеется система микроскопических трещин и пор непровара в начальном состоянии. При относительно небольшом росте внешних воздействий, а именно работы подвижной щеки дробилки, плотность микротрещин быстро растет. Трещины и поры увеличиваются в размерах, объединяются в один или несколько больших конгломератов и разрывают куски стекла на части - наступает разрушение структуры. Во время расширения трещин под действием раздавливания происходит концентрация напряжений. В результате, 
разрушение кусков стекла произойдет при небольшой величине среднего напряжения. У стекла происходит взрывоподобное разрушение формы за счет объемного напряжения.

В процессе работы использовалась дробилка ЩДД 1,6х2,5. После дробления стеклоотходы подавались на вибрационный грохот для процесса сегрегации материала. Ускорения, сообщаемые ситу, периодически изменялись по значению от нуля до максимума и направлению по синусоидальному закону.

Крупность материала, прошедшего процесс грохочения, составляла 30 мм. Далее продукт проходит стадию магнитной сепарации, во избежание попадания металлических включений. Для магнитной сепарации применялся подвесной магнит, который устанавливался над транспортерной лентой, обеспечивающей передвижение стекла.

После дробления, грохочения и магнитной сепарации, отходы стекольной промышленности смешивались с химическими добавками, в соотношении 40\% стекла и $60 \%$ добавок. Процесс смешивания осуществлялся в горшковой вращающейся стекловаренной печи, емкостью 150 л. Отходы стекла являются компонентом, обеспечивающим быстрое появление жидкой фазы, которая "впитывает" в себя химические добавки. Эти добавки выбирались по их влиянию на свойства стекол. Борная кислота и азотнокислый барий в составе стеклобоя были выбраны, с целью улучшения электрических характеристик, а именно диэлектрической постоянной, tg угла диэлектрических потерь. Борная кислота $\mathrm{H}_{3} \mathrm{~B}_{3}$, введенная в стекло, разлагается на борный ангидрид, который, в свою очередь, является ускорителем варки стекла. Кремнефтористый натрий $\mathrm{Na}_{2} \mathrm{SiF}_{6}$ в стекле вызывает появление жидкой фазы при более низких температурах. В связи с этим возрастает процесс силикатообразования и повышается электропроводность шихты. Азотнокислый калий $\mathrm{KNO}_{3}$ вводится в стекло для снижения его кристаллизации. Все введенные в стеклобой соединения являются плавнями и снижают температуру размягчения стекла.

Подбор процентного соотношения плавней производился по отношению к $\mathrm{SiO}_{2}$, как самому высокотемпературному оксиду, придающему стеклу особую тугоплавкость. Расчет вели на $100 \%$ массы.

В процентном соотношении он составил $40 \%$ стеклобоя, $14,4 \%$ азотнокислого калия KNO 3 $16,8 \%$ азотнокислого бария $\mathrm{Ba} \quad\left(\mathrm{NO}_{3}\right)_{2}, 19,2 \%$ борной кислоты $\mathrm{H}_{3} \mathrm{BO} 3$ и 9,6\% кремнефтористого натрия $\mathrm{Na}_{2} \mathrm{SiF}_{6}$. Процесс стекловарения можно разделить на 4 стадии:

1 стадия - силикатообразование.
Стеклобой с добавками в шихте претерпел ряд физических и химических изменений: в шихте закончились основные реакции в твердом состоянии, улетучилось большинство газообразных продуктов. К концу этой стадии шихта превратилась в спекшуюся массу. Силикатообразование проходило при температуре 800-900 C.

2 стадия - стеклообразование.

При дальнейшем нагревании спекшаяся масса начинала плавиться. Одновременно с плавлением происходило взаимное растворение силикатов и кремнезема. К концу этой стадии масса становилась прозрачной, однако, неоднородной по химическому составу. Стеклообразование протекало при температуре $1150-1200^{\circ} \mathrm{C}$. В процессе стекловарения на этих двух стадиях происходили следующие реакции:

$$
\begin{aligned}
& 2 \mathrm{KNO}^{3} \mathrm{~K}_{2} \mathrm{O}+2 \mathrm{NO}_{2}+\mathrm{l} / 2 \mathrm{O}_{2} \\
& \mathrm{Ba}\left(\mathrm{NO}_{3}\right)_{2} \mathrm{BaO}+\mathrm{NO}_{2} \\
& 4 \mathrm{H}_{3} \mathrm{BO}_{3} \longrightarrow 6 \mathrm{H}_{2} \mathrm{O}+2 \mathrm{~B}_{2} \mathrm{O}_{3} \\
& \mathrm{Na}_{2} \mathrm{SiF}_{6}+\mathrm{l} / 2 \mathrm{O}_{2} \longrightarrow \mathrm{NO}_{2}+\mathrm{SiF}_{4}+\mathrm{F}_{2}
\end{aligned}
$$

3 стадия - гомогенизация.

На этой стадии стекломассу выдерживали довольно длительное время при высоких температурах для выравнивания ее химического состава. Гомогенизации соответствовала температура $1400-1500^{\circ} \mathrm{C}$.

4 стадия - студка охлаждение.

Температуру стекломассы снижали на 200300 С для придания стеклу равномерной вязкости.

Расчет стадии дробления и измельчения стекла нового химического состава

Целью расчета схемы дробления является определение массы и выхода продуктов, и характеристика крупности продукта [4,с.39-54] . В качестве дробимого материала использовались отходы стекольной промышленности и разработанное стекло нового химического состава. Для расчета характеристики крупности необходимо знать приращение расчетного класса в операции дробления, подсчитываемое на основе допущения. Зерна, размер которых больше ширины загрузочной щели дробилки, дробятся одинаково, независимо от наличия или отсутствия в питании дробилки зерен, размер которых меньше ширины загрузочной щели. Наибольшая крупность зерен в классе, приращение которого необходимо определить, больше или равна ширине разгрузочной щели дробилки (d>i ). Приращение в рассматриваемом классе будет

$$
\Delta \mathrm{P}_{\mathrm{m}^{-}}{ }^{\mathrm{d}}=\mathrm{P}_{\mathrm{N}}{ }^{+\mathrm{d}} \mathrm{b}_{\mathrm{m}^{-}}{ }^{\mathrm{d}} \quad(\mathrm{d}>\mathrm{i}) \text {. }
$$


где b - содержание расчетного класса, мм;

п - индекс, показывающий номер продукта, поступающего в дробилку;

m - индекс, показывающий номер операции дробления.

Численные значения $\mathrm{b}_{\mathrm{m}^{-}}{ }^{\mathrm{d}}$ берутся по типовым характеристиками [6, с 29-34]. Общая масса загрузки стекла нового химического состава в щековую дробилку составляет 100 кг.

1) Определение массы продуктов 5 и 2. Эффективность грохочения для

любого класса крупности по определению равна

$$
\mathrm{E} 1^{\mathrm{d}}=\mathrm{P}_{2}^{\mathrm{d}} / \mathrm{P} 1^{\mathrm{d}}=\mathrm{Q}_{2} \beta_{2}{ }^{\mathrm{d}} / \mathrm{Q}_{1} \beta_{1}{ }^{\mathrm{d}},
$$

где

E1 - эффективность грохочения в операции по классу зерен размерами,

меньшими отверстий сит грохота;

$\mathrm{P} 1^{\mathrm{d}}$ - масса расчетного компонента в исходном питании, кг.;

$\mathrm{P}_{2}{ }^{\mathrm{d}}$ - масса расчетного компонента в продукте, кг.

Для класса - а формула эффективности имеет вид а - размер отверстий сит грохота, мм.

$$
\begin{aligned}
\mathrm{E} 1^{-\mathrm{a}}=\mathrm{P}_{2}^{-\mathrm{a}} / \mathrm{P}_{1}^{-\mathrm{a}} & =\mathrm{Q}_{2} \beta_{2}{ }^{-\mathrm{a}} / \mathrm{Q}_{1} \beta_{1}{ }^{-\mathrm{a}}=\mathrm{Q}_{2} * 1 / \mathrm{Q}_{1} * \beta_{1}^{-\mathrm{a}}= \\
& =\mathrm{Q}_{2 /} \mathrm{Q}_{1} * \beta_{1}^{-\mathrm{a}},
\end{aligned}
$$

где

Отсюда масса нижнего продукта

$$
\mathrm{Q}_{5}=\mathrm{Q}_{1} * \beta_{1}^{-30} \mathrm{E} 1^{-30}
$$

Q1- сухой вес идеального питания - 100 кг;

$\beta_{1}^{-30}$ - содержание массы класса крупностью от 0 до 30 мм, \%,

где Е - эффективность грохочения

$\mathrm{Ei}=85 \% \quad[7$, c. $43-48]$

$$
\mathrm{Q}_{5}=1000,30,85=25,5 \text { кг. , }
$$

Масса верхнего продукта

$$
\mathrm{Q}_{2}=\mathrm{Q}_{3}=\mathrm{Q} 1+\mathrm{Q} 5
$$

$\mathrm{Q}_{2}=\mathrm{Q}_{3}=100+25,5=125,5$ кг;

$\mathrm{Q} 1=\mathrm{Q}_{4}=100$ кг.

2) Определение характеристики крупности продукта 4

Масса класса - d продукте 4 равна массе этого класса в продукте 1 и приращении его в операции II.

$$
\mathrm{P}_{4}{ }^{\mathrm{d}}=\mathrm{P} 1{ }^{-\mathrm{d}}+\Delta \mathrm{PII}-^{\mathrm{d}}=\mathrm{Q} 1 \beta_{1}^{-\mathrm{d}}+\Delta \mathrm{PII}{ }^{-\mathrm{d}},
$$

где $\mathrm{P}_{4}$ - масса стекла на выходе, кг;

$\beta_{1}^{-\mathrm{d}}$ - содержание класса при загрузке, \%.

$$
\begin{gathered}
\mathrm{B}_{4}^{-\mathrm{d}}=\mathrm{P} 1-^{\mathrm{d}} / \mathrm{Q}_{4} / \mathrm{Q}_{4}=\mathrm{Q} 1 \beta_{1}^{-\mathrm{d}}+\Delta \mathrm{PII}^{-\mathrm{d}} / \mathrm{Q}_{4}= \\
=\beta_{1}^{-\mathrm{d}}+\Delta \mathrm{PII}^{-\mathrm{d}} / \mathrm{Q}_{1},
\end{gathered}
$$

так как $\mathrm{Q} 1=\mathrm{Q}_{4}$.

Приращение мелкого класса в стадии дробления при $\mathrm{d}>\mathrm{i}$

по формуле приращения будет

$\Delta \mathrm{PII}^{-\mathrm{d}}=\mathrm{P}_{5}^{+\mathrm{d}} \mathrm{bII}{ }^{-\mathrm{d}}$, поэтому формула примет вид

$$
\beta_{4}^{-\mathrm{d}}=\beta_{1}^{-\mathrm{d}}+\mathrm{P}_{5}^{+\mathrm{d}} \text { bII }{ }^{-\mathrm{d}} / \mathrm{Q}_{1}
$$

Аналогично первому случаю, когда $\mathrm{d}>\mathrm{i}$ масса класса $+\mathrm{d}$ в верхнем продукте

$$
\mathrm{P}_{5}^{+\mathrm{d}}=\mathrm{Q} 1\left(\beta_{1}^{+\mathrm{d}}-\beta_{1}^{-\mathrm{a}+\mathrm{d}} \mathrm{E}^{-\mathrm{a}+\mathrm{d}}\right),
$$

$$
\beta_{4}^{-d}=\beta_{1}^{-d}\left(\beta_{1}^{+d}+E^{-a+d}\right) b I I^{-d},
$$

Приближенно содержание

$$
\beta_{4}^{-d}=\beta_{1}^{-d}+\beta_{1}^{+d} \text { bII }{ }^{-d},
$$

$\beta_{4}^{-30}=\beta_{1}^{-\mathrm{d}}+\beta_{1}^{+30} \mathrm{bII}^{-\mathrm{d}}=0,3+0,70,94=0,96=96 \%$.

Выход продукта по отношению к исходному питанию

$\mathrm{Yn}=\mathrm{Q}_{\mathrm{n}} / \mathrm{Q} 1$;

$$
\mathrm{Y} 5=25,5 / 100=0,255=25,5 \% \text {; }
$$$$
\mathrm{Y} 2=\mathrm{Y} 3=\mathrm{Y} 1+\mathrm{Y} 5=1+25,5=26,5 \text {. }
$$

После получения фракции крупностью 30 мм стекло нового химического состава проходило операцию измельчения в струйной мельнице до крупности $-0,2+0,1$. 
Impact Factor ISRA (India) $\quad=\mathbf{1 . 3 4 4}$

Impact Factor ISI (Dubai, UAE) $=\mathbf{0 . 8 2 9}$

based on International Citation Report (ICR)

Impact Factor GIF (Australia) $\quad \mathbf{0} \mathbf{0 . 3 5 6}$
Impact Factor JIF $\quad=\mathbf{1 . 5 0 0}$

Impact Factor SIS (USA) $\quad=0.912$

Impact Factor РИНЦ (Russia) $=\mathbf{0 . 1 7 9}$

Impact Factor ESJI (KZ) $\quad=\mathbf{1 . 0 4 2}$
Расчет стадии измельчения

Схема измельчения стекла является схемой замкнутого цикла в одну стадию. Измельчение материала происходит до крупности, по заданной технологии получения слюдокерамических электронагревателей.

Расчет схемы сводится к определению массы и выхода продуктов.

1). Определение массы продуктов 2 и 5. Оптимальная циркулирующая нагрузка выбирается равной $120 \%$, [7, с.21-30].

Масса продуктов Q5=Q1-Сопт $=100 * 1,20=120$ кг; $\mathrm{Q} 1=\quad 100$ кг, $\mathrm{C}_{\text {oпr }^{-}}$оптимальная циркулирующая нагрузка; $\mathrm{Q} 2=\mathrm{O} 3=\mathrm{Q} 1 \mathrm{Q} 5=100+120=220$ кг; $\mathrm{Q}_{4}=\mathrm{Q} 1=100$ кг.

2). Выход продуктов находят по формуле $\mathrm{Yn}=\mathrm{Q}_{\mathrm{n}} / \mathrm{Q} 1$

$$
\begin{gathered}
\mathrm{Y}_{3}=\mathrm{Q}_{3} / \mathrm{Q} 1=220 / 100=2,2=22 \% \\
\mathrm{Y}_{5}=\mathrm{Q}_{5} / \mathrm{Q} 1=120 / 100=12 \% .
\end{gathered}
$$

Работа струйной мельницы заключается в том, что измельченный материал подается из бункера 1 питателем 2 в приемные камеры помольных эжекторов 4 или в стояк 7, соединяющий помольную камеру 6 с классификатором 9. Энергоноситель - воздух, под давлением 0,7-0,8Мпа, поступает по соплам 3 в приемные камеры помольных эжекторов. Истекающие из сопел струи энергоносителя расширяются и приобретают сверхзвуковую скорость. Частицы измельчаемого стекла, подсосанные в струи, вызывают в них ударные волны. При этом, газодинамическое сопротивление частиц возрастает почти на порядок, в результате они разгоняются до 100-150 м/с на сравнительно коротких участках в разгонных трубках 5. Ускоренные частицы, сталкиваясь, друг с другом с удвоенной скоростью, эффективно самоизмельчаются. Запыленный энергоноситель при движении по стояку разбавляется атмосферным воздухом, подсасываемым через поддувало, снабженное регулятором расхода. В пылеразделителе измельченный материал классифицируется. Классификатор обеспечивает выход фракции класса-0,2+0,1.

Грубая фракция выделяется из потока и по течкам 8 возвращается на домол в эжекторы, а тонкая осаждается в пылеосадителе 10 и через разгрузочное устройство 11 выводится из мельницы. Очищенный от пыли в фильтре 12 энергоноситель выбрасывается в атмосферу вентилятором 13. С целью установления закономерности распределения частиц стекла по размерам в зависимости от времени измельчения были проведены анализы гранулометрического состава проб измельченного стекла, Разработана и предложена математическая модель процесса оптимизации измельчения стекла по выходу класса крупности - 0,2 + 0,1 мм, имеющая экспоненциальный характер. Данный класс крупности исследовался в связи с его дальнейшим использованием в производстве слюдокерамических электронагревателей.

\section{References:}

1. Asnovich JZ, Zabyrina KI, Kolganov VA, Goreev BM (1979) Jelektro izoljacionnye materialy vysokoj nagrevostojkosti. Moscow: Jenergija, 1979. -pp.191-192.

2. Fedoseev GP (1961) Nekotorye osobennosti struktury mikaleksa // Sb.nauch.tehn.informacii IPRONIINEMETALLORUD.Moscow: 1961.- Vyp. 1. -pp.50-56.

3. Lomonosov MV (1940) Sbornik statej i materialov.- Moscow: Izd-vo AN SSSR, 1940. pp.560.

4. Fedoseev GP (1953) Mikaleks i ego svojstva i primenenie. Inf. soobshh. Tehn. Soveta i Tehn. Upr-ja ministerstva prom. strojmaterialov.Moscow: Promstrojizdat, 1953. -6 p.
5. Fedoseev GP (1965) Disperstnost' sljudy i dijelektricheskie poteri v mikalekse//Tam zhe.L.,1965.-Vyp.1. - pp.43-49.

6. Fedoseev GP (1967) Kratkij obzor proizvodstva mikaleksa za granicej // Tr.GIPRONIINEMETALLORUD.-L., $\quad$ 1967.Vyp.2. - pp.38-41.

7. Fedoseev GP (1967) Fiziko-himicheskaja obuslovlennost' udel'nogo jelektricheskogo poverhnostnogo soprotivlenija // Tam zhe.-L., 1967.-Vyp.2. -pp.1-12.

8. Fedoseev GP (1968) Svjaz' dijelektricheskih i mehanicheskih svojstv $\mathrm{v}$ mikalekse // Tr. GIPRONIINEMETALLORUD. - L., 1968.Vyp.Z.- pp.67-74. 
Impact Factor ISRA (India) $\quad=\mathbf{1 . 3 4 4}$

Impact Factor ISI (Dubai, UAE) $=\mathbf{0 . 8 2 9}$

based on International Citation Report (ICR)

Impact Factor GIF (Australia) $\quad \mathbf{0} \mathbf{0 . 3 5 6}$ $\begin{array}{lr}\text { Impact Factor JIF } & =\mathbf{1 . 5 0 0} \\ \text { Impact Factor SIS }(\text { USA) } & =\mathbf{0 . 9 1 2} \\ \text { Impact Factor PИНЦ (Russia) } & =\mathbf{0 . 1 7 9} \\ \text { Impact Factor ESJI }(\text { KZ }) & =\mathbf{1 . 0 4 2}\end{array}$

13. Gorelik VV (1973) Issledovanie nekotoryh fizicheskih svojst mikaleksa // Tr. GIPRONIINEMETALLORUD- L., 1973.Vyp.6. - pp.88-106.

14. Volkov KI, Zagibalov PN, Mecik AI (1971) Svojstvo, dobycha i pererabotka sljudy.I.:Vost-Sib.kn. izd-vo.1971. - pp.340.

15. Anosov VF, Borzov VG, Kurkip KS (1978) Ogneupornyj mikaleks, ego svojstva i primenenie pri rozlive aljuminija. Cvetnaja metallurgija.- 1978.-Vyp.12. -pp.58-60. 